\title{
Consumo e desempenho produtivo de cordeiros das raças Texel e Ideal terminados em confinamento com dietas contendo diferentes teores de casca de soja
}

\author{
Intake and productive performance of lambs of races Texel and \\ Ideal, in feedlot diets containing different levels of soybean hulls
}

\author{
Sérgio Carvalho ${ }^{*}$; Felipe Dotto Dias ${ }^{2}$; Cléber Cassol Pires ${ }^{3}$; \\ Tatiana Pfüller Wommer²; Rafael Sanches Venturini²; Mônica Feksa Frasson²; \\ Verônica Milanesi Pilecco²; Anderson Bortoluzi Moro²; Danielle Dias Brutti ${ }^{4}$
}

\section{Resumo}

\begin{abstract}
O presente experimento, conduzido no Laboratório de Ovinocultura da Universidade Federal de Santa Maria, teve como objetivo avaliar o efeito de níveis crescentes de substituição da silagem de sorgo por casca de soja sobre o consumo de nutrientes, o ganho de peso e as características de carcaça de cordeiros das raças Texel e Ideal, terminados em sistema de confinamento. Foram utilizados 40 cordeiros machos, não castrados, oriundos de parto simples, sendo 20 da raça Texel e 20 da raça Ideal, todos mantidos em baias individuais. Os tratamentos foram constituídos por duas raças (Texel e Ideal) e por quatro níveis de substituição de silagem de sorgo por casca de soja, sendo: 0; 33,5; 66,5 e 100\% de substituição. Os Texel foram abatidos com $32,88 \mathrm{~kg}$ e os Ideal com $27,51 \mathrm{~kg}$, correspondendo a $60 \%$ do peso vivo a maturidade das suas mães. Não foi observada interação entre raças e os níveis de casca de soja testados. $\mathrm{O}$ consumo de nutrientes aumentou linearmente nas diversas formas em que foi expresso, com o aumento de inclusão de casca de soja nas dietas. O ganho de peso diário foi influenciado de forma quadrática pela inclusão de casca de soja na dieta. O máximo ganho de peso de cordeiros, quando terminados em confinamento, é obtido quando se substitui silagem de sorgo por casca de soja em 56,7\%. Cordeiros da raça Texel terminados em sistema de confinamento apresentam maior ganho de peso diário e melhor conversão alimentar quando comparados a cordeiros da raça Ideal.
\end{abstract}

Palavras-chave: Alimentação, concentrado, ganho de peso, ovinos, volumoso

\begin{abstract}
This experiment, conducted in laboratory of sheep-raising of the Universidade Federal de Santa Maria, aimed to evaluate the effect of increasing levels of substitution of sorghum silage by soybean hulls on nutrient intake, weight gain and carcass traits lambs breeds Texel and Ideal, finished in feedlot. A total of 40 male lambs, not castrated, from simple birth, 20 and 20 of the breeds Texel and Ideal, all kept in individual pens. The treatments consisted of two breeds (Texel and Ideal) and four levels of substitution
\end{abstract}

\footnotetext{
${ }^{1}$ Prof. Adjunto, Dept $^{\mathrm{o}}$ de Zootecnia, Universidade Federal de Santa Maria, UFSM, Santa Maria, RS. E-mail: scarvalhoufsm@ hotmail.com

2 Discentes do Curso de Mestrado do Programa de Pós-Graduação em Zootecnia, UFSM, Santa Maria, RS. E-mail: dottofelipe@ yahoo.com.br; tatiwommer@hotmail.com; rs_venturini@hotmail.com; monicafrasson@zootecnista.com.br; vemilpilecco@ hotmail.com; andersondazoo@hotmail.com

3 Prof. Titular, Dept ${ }^{\circ}$ de Zootecnia, UFSM, Santa Maria, RS. E-mail: pirescleber@hotmail.com

${ }^{4}$ Discente do Curso de Graduação em Zootecnia, UFSM, Santa Maria, RS. E-mail: dani_brutti@hotmail.com

* Autor para correspondência
} 
of sorghum silage by soybean hulls, where: $0,33.5,66.5$ and $100 \%$ substitution. The Texel were slaughtered at $32.88 \mathrm{~kg}$ and Ideal at $27.51 \mathrm{~kg}$, corresponding to $60 \%$ of body weight at maturity of their mothers. There was no interaction between races and levels of soybean hulls tested. The nutrient intake increased linearly, in various forms in which it was expressed with increasing inclusion of soybean hulls in the diets. The daily weight gain was quadratically influenced by the inclusion of soybean hulls in the diet. The maximum weight gain of lambs when feedlot is obtained by replacing sorghum silage by soybean hulls at $56.7 \%$. Texel lambs finished in feedlot have a higher daily weight gain and better feed conversion when compared to the lambs breed Ideal.

Key words: Concentrate, feeding, roughage, sheep, weight gain

\section{Introdução}

Nos últimos anos tem ocorrido um aumento na demanda por carne ovina, sendo que a preferência dos consumidores tem sido por carne oriunda de animais jovens, o que tem levado aos ovinocultores a busca de novas tecnologias produtivas nesta atividade. Sabe-se também que no mercado de carne da atualidade, as transações comerciais tendem cada vez mais a serem realizadas com base na carcaça e suas características qualitativas e cada vez menos com base no que apresentam os animais vivos. Nesse sentido, na pecuária atual, pelas exigências impostas pelo mercado consumidor, não basta somente produzir mais quilogramas de carne a preços econômicos, mas sim, carcaças de melhor qualidade e uniformidade. Para suprir essa demanda do consumidor, o confinamento de cordeiros é uma prática que tem sido cada vez mais utilizada.

Nesse sistema, um alto potencial de desenvolvimento dos cordeiros pode ser alcançado quando se utilizam animais com potencial para ganho de peso e uma alimentação adequada, concomitantemente com uso adequado de tecnologias. Cordeiros criados em confinamento apresentam maior ganho de peso, em virtude da redução da carga parasitária e também por haver um menor gasto energético em função da redução da movimentação dos animais em busca de água e demais alimentos.

Contudo, em sistemas intensivos de produção de carne ovina, como o de confinamento, tornase cada vez mais importante a busca por alimento de qualidade para que a resposta animal seja satisfatória tornando esta atividade mais lucrativa. Segundo Restle e Vaz (1999), os alimentos podem representar cerca de $70 \%$ do custo total do confinamento, o que leva os produtores a buscarem alimentos alternativos, na tentativa de reduzir os custos de produção. Além disso, em períodos de baixa disponibilidade de forragens a busca por alternativas de ingredientes oriundos de processos agroindustriais tem se intensificado.

Entre as alternativas alimentares para uso na terminação de cordeiros em sistema de confinamento encontra-se a casca de soja, um subproduto obtido na indústria de óleo de soja e de produção de bicombustível, e que é produzida em grande quantidade no Brasil, pois conforme Zambom et al. (2001), constitui em torno de $2 \%$ do grão. Segundo o NRC (2001), a casca de soja é definida como um volumoso-concentrado, pois, tem a função fisiológica de fibra vegetal e funciona como um grão de cereal em termos de disponibilidade de energia. Em relação a sua composição química, a casca de soja possui alto teor de fibra em detergente neutro e fibra em detergente ácido, porém baixa quantidade de lignina (em torno de $2 \%$ ), o que pode resultar em uma digestibilidade de mais de $90 \%$.

Nesse sentido, devido a sua disponibilidade e as suas características nutricionais, associadas a praticidade no fornecimento aos animais, surge o interesse na utilização de casca de soja como fonte de volumoso a ser utilizado na dieta de cordeiros quando confinados em fase de terminação.

Outro fator que influencia no desempenho produtivo de cordeiros em confinamento é o 
grupo racial. Cordeiros de raças especializadas para a produção de carne, como a raça Texel, tem sido freqüentemente utilizados em sistemas de confinamento visando à obtenção de carne ovina de qualidade. Contudo, na região Sul do Brasil ainda existe uma base laneira, com um grande contingente do rebanho de ovinos da raça Ideal, onde os cordeiros têm sido destinados ao abate e para o mercado consumidor. Nesse sentido, estudos que comparem o desempenho e as características de carcaça de animais da raça Texel em relação aos da raça Ideal, são importantes que sejam conduzidos.

O objetivo deste trabalho foi avaliar o efeito de níveis crescentes de substituição da silagem de sorgo por casca de soja sobre o consumo de nutrientes, o ganho de peso e as características de carcaça de cordeiros das raças Texel e Ideal, terminados em sistema de confinamento.

\section{Material e Métodos}

O experimento foi conduzido no Laboratório de Ovinocultura do Departamento de Zootecnia da Universidade Federal de Santa Maria (UFSM), RS, no período compreendido entre outubro e dezembro de 2010. Foram utilizados 40 cordeiros machos, não castrados, oriundos de parto simples, sendo 20 da raça Texel e 20 da raça Ideal. Os animais foram confinados em baias individuais, totalmente cobertas, com piso ripado, aproximadamente 1,0 m acima do solo, com dimensão de $2 \mathrm{~m}^{2}$ por animal. Todas as baias eram providas de comedouros e bebedouros individuais, onde foi fornecida a alimentação e a água para os animais.

Os tratamentos foram constituídos por duas raças (Texel e Ideal) e por quatro níveis de substituição do alimento volumoso (silagem de sorgo) por casca de soja (\% da MS), sendo: 0; 33,5; 66,5 e 100\% de substituição.

Os cordeiros foram desmamados quando atingiram aproximadamente 50 dias de idade em média, e então distribuídos nos tratamentos. O volumoso utilizado foi silagem de sorgo (Sorghum bicolor (L.) Moench) e/ou casca de soja e o concentrado era constituído por milho desintegrado, farelo de soja e mistura mineral. As dietas foram formuladas para serem isoprotéicas, de acordo com o NRC (2007), sendo que a relação entre silagem de sorgo e casca de soja variou de acordo com o tratamento, em base da matéria seca (MS), de maneira a se atingir o nível de casca de soja, como alimento volumoso, pretendido para a dieta experimental (Tabela 1).

$\mathrm{O}$ alimento foi fornecido, ad libitum, duas vezes ao dia, sendo os horários de arraçoamento às 8:00 e 17:00 horas. A quantidade oferecida era ajustada em função da sobra observada diariamente, sendo que esta deveria ser $10 \%$ da quantidade oferecida no dia anterior, de modo a garantir o consumo voluntário máximo dos animais.

O período experimental foi precedido de um período de 10 dias para adaptação dos animais as condições de instalações, alimentação e manejo. $\mathrm{O}$ ensaio de alimentação iniciou após o período de adaptação, estendendo-se até o momento em que cada cordeiro atingiu o peso de abate préestabelecido em $32 \mathrm{~kg}$ de peso vivo para os animais da raça Texel e $27 \mathrm{~kg}$ de peso vivo para os animais da raça Ideal, correspondendo a $60 \%$ do peso vivo a maturidade das suas mães. Os cordeiros foram pesados no início e final da fase experimental, após jejum de sólidos de 14 horas, sendo que para um melhor acompanhamento do desempenho, foram realizadas pesagens intermediárias a cada 7 dias.

As amostras de alimentos e sobras foram pré-secas em estufa ventilada a $55^{\circ} \mathrm{C}$ por aproximadamente 72 horas e, posteriormente, moídas em moinho tipo "Willey" com peneira de $1 \mathrm{~mm}$. A determinação dos teores de matéria seca (MS) foi realizada por secagem em estufa a $105^{\circ} \mathrm{C}$ durante 24 horas, e de cinzas por incineração em mufla a $550^{\circ} \mathrm{C}$ por duas horas (SILVA, 1998). A fibra em detergente neutro (FDN) e fibra em detergente ácido (FDA) foram realizadas segundo metodologia descrita por 
Senger et al. (2008). O teor de nitrogênio total (N) foi determinado pelo método Kjeldahl (AOAC, 1990), modificado segundo Kozloski et al. (2003). Para conversão dos valores de $\mathrm{N}$ em proteína bruta (PB) foi utilizado o fator de correção de 6,25. A determinação dos teores de extrato etéreo (EE) foi realizada em sistema de refluxo de éter (Soxtherm, Gerhardt, Alemanha) a $180^{\circ} \mathrm{C}$ durante duas horas. Os teores de carboidratos totais (CHT) foram calculados pela equação CHT $(\%)=100-(\%$ PB $+\% \mathrm{EE}+\% \mathrm{CIN})$, e os teores de carboidratos nãofibrosos (CNF), pela diferença de CHT - FDN.

Tabela 1. Proporção dos ingredientes (\%MS) e composição bromatológica das dietas experimentais.

\begin{tabular}{|c|c|c|c|c|}
\hline & \multicolumn{4}{|c|}{$\%$ de casca de soja } \\
\hline & 0 & 33,5 & 66,5 & 100 \\
\hline \multicolumn{5}{|c|}{ Proporção dos ingredientes (\%MS) } \\
\hline Silagem de sorgo & 50,00 & 33,25 & 16,75 & 0,00 \\
\hline Casca de soja & 0,00 & 16,75 & 33,25 & 50,00 \\
\hline Milho quebrado & 22,96 & 25,63 & 28,27 & 30,95 \\
\hline Farelo de soja & 26,18 & 23,53 & 20,92 & 18,27 \\
\hline Calcário calcítico & 0,86 & 0,84 & 0,81 & 0,78 \\
\hline \multicolumn{5}{|c|}{ Composição bromatológica (\%MS) } \\
\hline MS & 57,29 & 68,02 & 78,58 & 89,31 \\
\hline MO & 95,68 & 95,82 & 95,95 & 96,08 \\
\hline PB & 17,00 & 17,00 & 17,00 & 17,00 \\
\hline $\mathrm{EE}$ & 4,37 & 4,71 & 5,04 & 5,38 \\
\hline FDN & 38,87 & 38,40 & 37,94 & 37,47 \\
\hline CHT & 73,45 & 73,27 & 73,10 & 72,92 \\
\hline CNE & 34,59 & 34,87 & 35,16 & 35,45 \\
\hline CIN & 4,32 & 4,18 & 4,05 & 3,92 \\
\hline NDT & 69,99 & 72,09 & 74,17 & 76,28 \\
\hline EL & 1,60 & 1,65 & 1,70 & 1,75 \\
\hline $\mathrm{Ca}$ & 0,55 & 0,55 & 0,55 & 0,55 \\
\hline $\mathrm{P}$ & 0,31 & 0,30 & 0,28 & 0,26 \\
\hline
\end{tabular}

Fonte: Elaboração dos autores.

Ao atingirem o peso de abate, os animais eram pesados após jejum de sólidos por 14 horas, insensibilizados mediante atordoamento e abatidos mediante sangria. Após cada abate, a carcaça foi pesada individualmente e em seguida resfriada por 24 horas em câmara frigorífica, a uma temperatura de $2^{\circ}$ C. Foram observadas as seguintes características em relação às carcaças dos cordeiros. Peso da carcaça quente (PCQ): obtida através de pesagem logo após o abate; Peso da carcaça fria (PCF): tomado após a permanência de 24 horas em câmara fria a uma temperatura média de $2^{\circ} \mathrm{C}$; Índice de quebra ao resfriamento (IQ): cálculo através da diferença entre o PCQ e PCF; Rendimento de carcaça quente (RCQ): relação percentual entre peso vivo ao abate e PCQ; Rendimento de carcaça fria (RCF): relação percentual entre peso vivo ao abate e PCF.

$\mathrm{O}$ delineamento experimental utilizado foi $\mathrm{o}$ inteiramente casualizado, em esquema fatorial $2 \mathrm{x}$ 4 (duas raças x 4 níveis de substituição de silagem de sorgo por casca de soja), com cinco repetições por tratamento. Foi testado e efeito do nível de casca de soja, da raça e da interação casca x raça, através de análise de variância e teste $\mathrm{F}$, no nível 
de $5 \%$ de significância. $O$ efeito do percentual de casca foi analisado através de análise de regressão. As análises foram realizadas utilizando-se o pacote estatístico SAS (2001).

\section{Resultados e Discussão}

Não foi observada interação entre a raça e os níveis de casca de soja testados. Portanto, os resultados são apresentados de forma independente.

O consumo de nutrientes aumentou linearmente, nas diversas formas em que foi expresso, com o aumento de inclusão de casca de soja nas dietas (Tabela 2). Esse resultado pode ser explicado devido à melhoria da palatabilidade, por ocasião da inclusão da casca de soja, e o aumento da taxa de passagem da casca de soja em relação à silagem de sorgo como conseqüência do menor tamanho de partícula e maior digestibilidade da fração fibrosa do alimento. Anderson et al. (1988), relataram que a casca de soja apresenta em torno de $74 \%$ de FDN na matéria seca, sendo $47 \%$ composto de celulose e $23 \%$ de hemicelulose, o que confere alta digestibilidade da fração fibrosa a este subproduto. Já em relação a sua composição química, a casca de soja possui alto teor em fibra em detergente neutro e fibra em detergente ácido, porém baixa quantidade de lignina (em torno de $2 \%$ ), o que pode resultar em uma digestibilidade in vitro de mais de $90 \%$.

Tabela 2. Consumos médios de matéria seca (CMS), matéria orgânica (CMO), proteína bruta (CPB), extrato etéreo (CEE), fibra em detergente neutro (CFDN), carboidratos totais (CCHT) e carboidratos não fibrosos (CCNF), para cordeiros Texel e Ideal, de acordo com os teores de substituição de silagem de sorgo por casca de soja.

\begin{tabular}{|c|c|c|c|c|c|c|c|}
\hline & \multicolumn{4}{|c|}{$\%$ de casca de soja } & \multirow{2}{*}{ Equação de regressão ${ }^{1}$} & \multirow{2}{*}{$\mathrm{R}^{2}$} & \multirow{2}{*}{$\mathrm{P}>\mathrm{F}$} \\
\hline & 0 & 33,5 & 66,5 & 100 & & & \\
\hline \multicolumn{8}{|c|}{$\mathrm{kg} / \mathrm{dia}$} \\
\hline $\mathrm{CMS}$ & 0,697 & 0,840 & 0,889 & 0,930 & $\hat{\mathrm{Y}}=0,72928+0,00222 \mathrm{CS}$ & 0,41 & 0,0001 \\
\hline $\mathrm{CMO}$ & 0,666 & 0,804 & 0,853 & 0,894 & $\hat{Y}=0,69647+0,00217 C S$ & 0,43 & 0,0001 \\
\hline CPB & 0,138 & 0,159 & 0,161 & 0,172 & $\hat{Y}=0,14212+0,00031 C S$ & 0,27 & 0,0007 \\
\hline CEE & 0,035 & 0,043 & 0,048 & 0,054 & $\hat{Y}=0,03584+0,00019 C S$ & 0,64 & 0,0001 \\
\hline CFDN & 0,217 & 0,279 & 0,326 & 0,344 & $\hat{Y}=0,22798+0,00127 C S$ & 0,61 & 0,0001 \\
\hline $\mathrm{CCHT}$ & 0,488 & 0,594 & 0,643 & 0,675 & $\hat{\mathrm{Y}}=0,51001+0,00181 \mathrm{CS}$ & 0,49 & 0,0001 \\
\hline CCNF & 0,271 & 0,316 & 0,323 & 0,330 & $\hat{Y}=0,28338+0,00054 C S$ & 0,22 & 0,0029 \\
\hline \multicolumn{8}{|c|}{$\% \mathrm{PV}$} \\
\hline $\mathrm{CMS}$ & 2,69 & 3,31 & 3,52 & 3,68 & $\hat{\mathrm{Y}}=2,83623+0,00941 \mathrm{CS}$ & 0,42 & 0,0001 \\
\hline $\mathrm{CMO}$ & 2,57 & 3,17 & 3,38 & 3,54 & $\hat{Y}=2,70950+0,00920 C S$ & 0,43 & 0,0001 \\
\hline $\mathrm{CPB}$ & 0,53 & 0,63 & 0,64 & 0,68 & $\hat{\mathrm{Y}}=0,55355+0,00135 \mathrm{CS}$ & 0,31 & 0,0002 \\
\hline CEE & 0,13 & 0,17 & 0,19 & 0,21 & $\hat{\mathrm{Y}}=0,13924+0,00077 \mathrm{CS}$ & 0,65 & 0,0001 \\
\hline CFDN & 0,84 & 1,10 & 1,29 & 1,36 & $\hat{Y}=0,88403+0,00528 \mathrm{CS}$ & 0,56 & 0,0001 \\
\hline $\mathrm{CCHT}$ & 1,88 & 2,34 & 2,54 & 2,67 & $\hat{Y}=1,98289+0,00762 C S$ & 0,48 & 0,0001 \\
\hline CCNF & 1,04 & 1,25 & 1,28 & 1,31 & $\hat{\mathrm{Y}}=1,10374+0,00236 \mathrm{CS}$ & 0,25 & 0,0013 \\
\hline \multicolumn{8}{|c|}{$\mathbf{g} / \mathbf{k g} \mathbf{P V}, 75$} \\
\hline $\mathrm{CMS}$ & 60,63 & 74,17 & 78,70 & 82,38 & $\hat{\mathrm{Y}}=63,7044+0,20686 \mathrm{CS}$ & 0,47 & 0,0001 \\
\hline $\mathrm{CMO}$ & 57,94 & 71,01 & 75,46 & 79,22 & $\hat{Y}=60,8548+0,20248 C S$ & 0,48 & 0,0001 \\
\hline СРB & 11,99 & 14,02 & 14,39 & 15,19 & $\hat{\mathrm{Y}}=12,4284+0,02957 \mathrm{CS}$ & 0,34 & 0,0001 \\
\hline CEE & 3,04 & 3,83 & 4,21 & 4,82 & $\hat{Y}=3,12508+0,01708 \mathrm{CS}$ & 0,70 & 0,0001 \\
\hline CFDN & 18,82 & 24,62 & 28,80 & 30,48 & $\hat{\mathrm{Y}}=19,8743+0,11665 \mathrm{CS}$ & 0,60 & 0,0001 \\
\hline CCHT & 42,41 & 52,46 & 56,88 & 59,78 & $\hat{Y}=44,5434+0,16784 C S$ & 0,53 & 0,0001 \\
\hline $\mathrm{CCNF}$ & 23,58 & 27,95 & 28,58 & 29,22 & $\hat{\mathrm{Y}}=24,7796+0,05163 \mathrm{CS}$ & 0,27 & 0,0007 \\
\hline
\end{tabular}

${ }^{1} \mathrm{CS}=$ Nível de substituição do volumoso da dieta por casca de soja.

Fonte: Elaboração dos autores. 
O resultado obtido no presente estudo é corroborado por Morais et al. (2007), que observaram aumento linear do consumo de matéria seca em $\mathrm{kg} / \mathrm{dia}$, \% de peso vivo e em $\mathrm{g} / \mathrm{kg} \mathrm{PV}^{0,75} \mathrm{com}$ a inclusão de $0 \%, 12,5 \%, 25 \%$ e $37,5 \%$ de casca de soja em substituição ao feno de coastcross na dieta de borregas confinadas. Os autores atribuíram o aumento do consumo a maior taxa de passagem da dieta, explicada pela maior digestibilidade da FDN e ao menor tamanho de partícula da casca de soja quando comparada ao feno de coastcross. Da mesma forma Araujo et al. (2008), observaram aumento linear no consumo de matéria seca à medida que a casca de soja foi incluída na dieta em um experimento avaliando o efeito da substituição da FDN de feno de coastcross pela FDN da casca de soja na alimentação de cordeiros da raça Santa Inês.

Em relação as raças, não foi constatada diferença significativa para estes parâmetros, quando referenciados em relação ao tamanho metabólico dos animais, o que já era esperado, pois o peso metabólico homogeneíza os animais por superfície corporal, retirando o efeito do peso vivo (Tabela 3). Por outro lado, quando expressos em $\mathrm{kg} / \mathrm{dia}$, constatou-se diferença significativa, sendo que os cordeiros da raça Texel apresentaram um maior consumo de MS e de nutrientes em relação aos cordeiros da raça Ideal. Uma vez que os cordeiros da raça Texel apresentaram peso vivo inicial e peso vivo ao abate superior aos da raça Ideal, o resultado obtido pode ser explicado por esse aspecto, pois conforme Pereira et al. (2003), em geral, a ingestão de matéria seca eleva-se com o aumento do peso vivo do animal. De forma inversa, quando o consumo foi expresso em \% do PV, verificou-se superioridade para os cordeiros da raça Ideal, o que pode ser relacionado a uma maior proporção de trato gastrintestinal de cordeiros da raça Ideal quando comparados aos da raça Texel, o que proporciona uma maior capacidade de ingestão.

Tabela 3. Consumos médios de matéria seca $(\mathrm{CMS})$, matéria orgânica $(\mathrm{CMO})$, proteína bruta $(\mathrm{CPB})$, extrato etéreo (CEE), fibra em detergente neutro (CFDN), carboidratos totais (CCHT) e carboidratos não fibrosos (CCNF), de acordo com a raça dos cordeiros.

\begin{tabular}{|c|c|c|c|c|}
\hline & \multicolumn{2}{|c|}{ Raça } & \multirow[b]{2}{*}{ Média } & \multirow[b]{2}{*}{$\mathrm{P}>\mathrm{F}$} \\
\hline & Ideal & Texel & & \\
\hline \multicolumn{5}{|c|}{ kg/dia } \\
\hline CMS & $0,796^{\mathrm{b}}$ & $0,887^{\mathrm{a}}$ & 0,843 & 0,0011 \\
\hline $\mathrm{CMO}$ & $0,763^{\mathrm{b}}$ & $0,850^{\mathrm{a}}$ & 0,808 & 0,0011 \\
\hline CPB & $0,149^{b}$ & $0,167^{\mathrm{a}}$ & 0,158 & 0,0009 \\
\hline CEE & $0,043^{b}$ & $0,048^{\mathrm{a}}$ & 0,045 & 0,0006 \\
\hline CFDN & $0,281^{b}$ & $0,305^{\mathrm{a}}$ & 0,293 & 0,0198 \\
\hline $\mathrm{CCHT}$ & $0,571^{b}$ & $0,633^{\mathrm{a}}$ & 0,603 & 0,0015 \\
\hline CCNF & $0,292^{b}$ & $0,329^{\mathrm{a}}$ & 0,311 & 0,0011 \\
\hline \multicolumn{5}{|c|}{$\%$ PV } \\
\hline CMS & $3,49^{\mathrm{a}}$ & $3,15^{\mathrm{b}}$ & 3,32 & 0,0185 \\
\hline $\mathrm{CMO}$ & $3,35^{\mathrm{a}}$ & $3,02^{\mathrm{b}}$ & 3,18 & 0,0183 \\
\hline СРB & $0,65^{\mathrm{a}}$ & $0,59^{\mathrm{b}}$ & 0,62 & 0,0277 \\
\hline CEE & $0,19^{a}$ & $0,17^{\mathrm{b}}$ & 0,18 & 0,0228 \\
\hline CFDN & $1,23^{\mathrm{a}}$ & $1,08^{\mathrm{b}}$ & 1,15 & 0,0227 \\
\hline CCHT & $2,50^{\mathrm{a}}$ & $2,25^{\mathrm{b}}$ & 2,37 & 0,0153 \\
\hline CCNF & $1,28^{\mathrm{a}}$ & $1,17^{\mathrm{b}}$ & 1,22 & 0,0338 \\
\hline
\end{tabular}


Continua ...

\begin{tabular}{|c|c|c|c|c|}
\hline \multicolumn{5}{|c|}{$\mathrm{g} / \mathrm{kg} \mathrm{PV}^{0,75}$} \\
\hline CMS & 76,19 & 72,53 & 74,31 & 0,2922 \\
\hline $\mathrm{CMO}$ & 73,04 & 69,52 & 71,23 & 0,2909 \\
\hline CPB & 14,24 & 13,66 & 13,94 & 0,3796 \\
\hline CEE & 4,10 & 3,91 & 4,00 & 0,3624 \\
\hline CFDN & 26,82 & 24,94 & 25,86 & 0,2046 \\
\hline $\mathrm{CCHT}$ & 54,61 & 51,76 & 53,15 & 0,2506 \\
\hline $\mathrm{CCNF}$ & 27,99 & 26,89 & 27,43 & 0,4033 \\
\hline
\end{tabular}

Fonte: Elaboração dos autores.

O ganho de peso diário foi influenciado de forma quadrática pela inclusão de casca de soja na dieta (Tabela 4). A partir da equação de regressão, estima- se que o máximo ganho de peso, correspondendo a $0,306 \mathrm{~kg} / \mathrm{dia}$, pode ser obtido com a substituição de silagem de sorgo por casca de soja ao nível de $56,7 \%$.

Tabela 4. Valores médios para peso vivo inicial (PVI), peso vivo ao abate (PVA), ganho de peso diário (GPD), conversão alimentar (CA), peso de carcaça quente (PCQ), peso de carcaça fria (PCF), rendimento de carcaça quente (RCQ), rendimento de carcaça fria (RCF) e índice de quebra ao resfriamento (IQ), para cordeiros Texel e Ideal, de acordo com os teores de substituição de silagem de sorgo por casca de soja.

\begin{tabular}{|c|c|c|c|c|c|c|c|}
\hline & \multicolumn{4}{|c|}{$\%$ de casca de soja } & \multirow{2}{*}{ Equação de regressão $^{1}$} & \multirow{2}{*}{$\mathrm{R}^{2}$} & \multirow{2}{*}{$\mathrm{P}>\mathrm{F}$} \\
\hline & 0 & 33,5 & 66,5 & 100 & & & \\
\hline PVI (kg) & 21,42 & 19,96 & 20,26 & 20,06 & $\hat{\mathrm{Y}}=20,40$ & ----- & 0,4328 \\
\hline PVA $(\mathrm{kg})$ & 30,28 & 30,51 & 30,12 & 30,15 & $\hat{\mathrm{Y}}=30,26$ & ---- & 0,8475 \\
\hline GPD $(\mathrm{kg})$ & 0,201 & 0,293 & 0,299 & 0,247 & $\hat{\mathrm{Y}}=0,2030+0,00363 \mathrm{CS}-0,000032 \mathrm{CS}^{2}$ & 0,29 & 0,0010 \\
\hline $\mathrm{CA}$ & 3,67 & 2,90 & 2,95 & 3,71 & $\hat{\mathrm{Y}}=3,6606-0,03356 \mathrm{CS}+0,00034 \mathrm{CS}^{2}$ & 0,35 & 0,0001 \\
\hline PCQ (kg) & 13,42 & 13,71 & 13,58 & 14,09 & $\hat{\mathrm{Y}}=13,70$ & ----- & 0,5654 \\
\hline PCF (kg) & 12,96 & 13,27 & 13,15 & 13,65 & $\hat{Y}=13,27$ & ----- & 0,5502 \\
\hline RCQ (\%) & 44,02 & 44,66 & 44,85 & 46,45 & $\hat{\mathrm{Y}}=45,02$ & ----- & 0,1539 \\
\hline RCF (\%) & 42,52 & 43,23 & 43,41 & 45,00 & $\hat{\mathrm{Y}}=43,57$ & ----- & 0,1448 \\
\hline IQ (\%) & 3,44 & 3,23 & 3,19 & 3,14 & $\hat{\mathrm{Y}}=3,24$ & ----- & 0,2529 \\
\hline
\end{tabular}

Fonte: Elaboração dos autores.

Os valores obtidos para ganho de peso diário deste estudo estão de acordo com o esperado, visto que as dietas experimentais foram formuladas para ganho entre 200 e $300 \mathrm{~g} /$ dia, segundo o NRC (1985). Morais et al. (2007), trabalhando com borregas na fase de crescimento alimentadas com $0 \%, 12,5 \%$, $25 \%$ e $37,5 \%$ de substituição do volumoso da dieta (feno de coastcross) por casca de soja, constataram que o ganho de peso diário aumentou linearmente, variando de 113 a $187 \mathrm{~g} /$ dia. Esses autores concluíram que a casca de soja utilizada em até $37,5 \%$ da matéria seca total da dieta para borregas na fase de crescimento melhora o ganho de peso dos animais, valor esse que está abaixo do valor de $56,7 \%$ obtido no presente estudo como nível ótimo de substituição.

A conversão alimentar também foi influenciada de forma quadrática, resultado esse que pode ser relacionado aquele obtido para a variável ganho de peso diário. Deve-se destacar que, conforme Ribeiro 
(1996), a CA de cordeiros pode ser de 1:1 no período inicial de amamentação, pode baixar para 10:1 no desmame, se eles estiverem em pastagens pobres, e na terminação, se forem alimentados com rações de boa qualidade, pode chegar a 3:1. O aspecto de que os cordeiros, independente do tratamento, foram alimentados em confinamento com dietas de boa qualidade e que foram formuladas para atender as exigências nutricionais dos animais, o que resultou em alta taxa de crescimento, explica a boa CA obtida.

O valor médio observado para $\mathrm{CA}$, de 3,30 é semelhante ao encontrado por Brochier e Carvalho (2008), que trabalharam com proporções de substituição de $0 \%, 33 \%, 66 \%$ e $100 \%$ do alimento concentrado por resíduo úmido de cervejaria, e verificaram valor médio para ganho de peso diário de 0,232 kg e CA média de 3,46:1, em cordeiros da raça Texel. Contudo, cabe salientar que esses autores trabalharam com silagem de milho como alimento volumoso, na proporção de $50 \%$ da dieta total. Resultados semelhantes aos obtidos nesse estudo foram também verificados por Susin, Rocha e Pires (2000), que utilizaram uma dieta com $80 \%$ de concentrado e observaram para cordeiros uma conversão alimentar de 3,89.

Em relação aos dados de peso de carcaça quente e fria, rendimento de carcaça quente e fria e índice de quebra ao resfriamento (Tabela 4), verificase que as variáveis não foram influenciadas pelo nível de inclusão de casca de soja, o que pode ser explicado pelo fato dos animais serem abatidos com peso semelhante.

Quanto ao índice de quebra ao resfriamento, deve-se enfatizar que esse índice indica o percentual de peso que é perdido durante o resfriamento da carcaça, em função de alguns fatores, como perda de umidade e reações químicas que ocorrem no músculo. Assim, quanto menor esse percentual, maior é a probabilidade da carcaça ter sido manejada e armazenada de maneira adequada. Neste experimento, o índice de quebra ao resfriamento não foi influenciado pela proporção de inclusão de casca de soja, apresentando valor médio de 3,24\%, um pouco superior ao resultado de Brito et al. (2005), os quais citam que de forma geral, os valores para os índices de quebra ao resfriamento estão em torno de $2,5 \%$.

Cordeiros da raça Texel terminados em confinamento apresentam maior ganho de peso diário e melhor conversão alimentar quando comparados a cordeiros da raça Ideal (Tabela 5). O fato de que os cordeiros da raça Texel são especializados para produção de carne, enquanto que os da raça Ideal são especializados para a produção de lã, explica esse resultado.

Tabela 5. Valores médios para peso vivo inicial (PVI), peso vivo ao abate (PVA), ganho de peso diário (GPD), conversão alimentar (CA), peso de carcaça quente (PCQ), peso de carcaça fria (PCF), rendimento de carcaça quente (RCQ), rendimento de carcaça fria (RCF) e índice de quebra ao resfriamento (IQ), de acordo com a raça dos cordeiros.

\begin{tabular}{|c|c|c|c|c|}
\hline & \multicolumn{2}{|c|}{ Raça } & \multirow{2}{*}{ Média } & \multirow{2}{*}{$\mathrm{P}>\mathrm{F}$} \\
\hline & Ideal & Texel & & \\
\hline PVI (kg) & $17,75^{\mathrm{b}}$ & $22,92^{\mathrm{a}}$ & 20,40 & 0,0001 \\
\hline PVA (kg) & $27,51^{\mathrm{b}}$ & $32,88^{\mathrm{a}}$ & 30,26 & 0,0001 \\
\hline GPD (kg) & $0,239^{\mathrm{b}}$ & $0,282^{\mathrm{a}}$ & 0,261 & 0,0116 \\
\hline CA & 3,43 & 3,17 & 3,30 & 0,0858 \\
\hline PCQ (kg) & $11,54^{\mathrm{b}}$ & $15,76^{\mathrm{a}}$ & 13,70 & 0,0001 \\
\hline PCF (kg) & $11,14^{b}$ & $15,29^{\mathrm{a}}$ & 13,27 & 0,0001 \\
\hline RCQ (\%) & $41,94^{b}$ & $47,94^{\mathrm{a}}$ & 45,02 & 0,0001 \\
\hline RCF (\%) & $40,47^{\mathrm{b}}$ & $46,51^{\mathrm{a}}$ & 43,57 & 0,0001 \\
\hline IQ (\%) & $3,51^{\mathrm{a}}$ & $2,99^{\mathrm{b}}$ & 3,24 & 0,0018 \\
\hline
\end{tabular}

Fonte: Elaboração dos autores. 
Furusho-Garcia et al. (2000) trabalhando com animais de diferentes grupos genéticos (Texel x Bergamácia; Texel x Santa Inês; Santa Inês) concluíram que os animais provenientes do cruzamento industrial, utilizando a raça Texel como raça paterna, obtiveram desempenho superior em comparação aos animais Santa Inês puros. Pires et al. (1999), afirmam que os cordeiros Cruzas F1 (1/2 Texel + 1/2 Ideal) e cordeiros F2 (3/4 Texel + 1/4 Ideal) apresentam maior ganho de peso médio diário do que cordeiros da raça Ideal, quando abatidos à mesma maturidade.

Em relação ao peso de carcaça quente e fria, rendimento de carcaça quente e fria e índice de quebra ao resfriamento, observa-se que os cordeiros da raça Texel apresentaram resultados superiores aos da raça Ideal em todas estas variáveis. O resultado pode ser justificado para os pesos de carcaça quente e de carcaça fria pelo peso superior de abate préestabelecido em $32 \mathrm{~kg}$ de peso vivo para os animais da raça Texel e $27 \mathrm{~kg}$ de peso vivo para os animais da raça Ideal, correspondendo a $60 \%$ do peso vivo a maturidade das suas mães. Quanto ao rendimento de carcaça quente e de carcaça fria, o resultado pode ser explicado pelo aspecto de que animais da raça Texel apresentam uma melhor conformação de carcaça do que os animais da raça Ideal, além de apresentarem uma menor proporção de pele e de trato gastrintestinal, variáveis essas que mais influencia no rendimento de carcaça dos animais.

\section{Conclusões}

O aumento da substituição de silagem de sorgo por casca de soja na dieta de cordeiros em sistema de confinamento proporciona um aumento do consumo de nutrientes por parte dos animais. Podese recomendar o nível de substituição de silagem de sorgo por casca de soja na proporção de $56,7 \%$, pois é aquele que proporciona o melhor ganho de peso dos cordeiros confinados.

Cordeiros da raça Texel e da raça Ideal apresentam consumo de nutrientes semelhantes por unidade de tamanho metabólico. Porém, cordeiros da raça Texel apresentam um melhor ganho de peso diário e maior rendimento de carcaça quente, rendimento de carcaça fria e melhor índice de quebra ao resfriamento que animais da raça Ideal, quando terminados em confinamento.

\section{Comitê de Ética e Biossegurança}

O presente trabalho faz parte do projeto de pesquisa aprovado pelo Comitê de Ética no Uso de Animais da Universidade Federal de Santa Maria (CEUA-UFSM), protocolo: 006/2011.

\section{Referências}

ANDERSON, S. J.; MERRIL, J. K.; McDONNELL, M. L.; KLOPFENSTEIN, T. J. Digestibility and utilization of mechanically processed soybean hulls by lambs and steers. Journal of Animal Science, Champaign, v. 66, n. 11, p. 2965-2976, 1988.

ARAUJO, R. C.; PIRES, A. V.; SUSIN, I.; URANO, F. S.; MENDES, C. Q.; RODRIGUES, G. H.; PACKER, I. U. Apparent digestibility of diets with combinations of soybean hulls and coastcross (Cynodon sp.) hay offered to ram lambs. Scientia Agricola, Piracicaba, v. 65, n. 6, p. 581-588, 2008.

ASSOCIATION OF OFFICIAL ANALYTICAL CHEMISTS - AOAC. Official methods of analysis. 15. ed. Washington, DC: Association of Official Analytical Chemistry, 1990. v. 1, 1422 p.

BRITO, R. A. M.; DIAS, M. J.; DIAS, D. S. O.; NUNES, I. A.; MESQUITA, A. J.; NOGUEIRA, A. T.; TONIN, M. C.; HENRIQUE, C.; MASTALOZZI, H. Características da carcaça e composição centesimal da carne de borregos de dois genótipos criados em confinamento. In: CONGRESSO DE PESQUISA, ENSINO E EXTENSÃO, 2., 2005, Goiânia. Anais... Goiânia: UFG, 2005. CD-ROM.

BROCHIER, M. A.; CARVALHO, S. Consumo, ganho de peso e análise econômica da terminação de cordeiros em confinamento com dietas contendo diferentes proporções de resíduo úmido de cervejaria. Arquivo Brasileiro de Medicina Veterinária e Zootecnia, Belo Horizonte, v. 60, n. 5, p. 1205-1212, 2008.

FURUSHO-GARCIA, I. F.; PEREZ, J. R. O.; TEIXEIRA, J. C.; BARBOSA, C. M. P. Desempenho de cordeiros Texel x Bergamácia, Texel x Santa Inês e Santa Inês 
puros,terminados em confinamento, alimentados com casca de café como parte da dieta. Revista Brasileira de Zootecnia, Viçosa, MG, v. 29, n. 2, p. 564-572, 2000.

KOZLOSKI, G. V.; PEROTONI, J.; CIOCCA, M. L. S.; ROCHA, J. B. T.; RAISER, A. G.; SANCHEZ, L. M. B. Potential nutritional assessment of dwarf elephant grass (Pennisetum purpureum Schum. Mott) by chemical composition, digestion and net portal flux of oxygen in cattle. Animal Feed Science and Technology, Amsterdam, v. 104, n. 1-4, p. 29-40, 2003.

MORAIS, J. B.; SUSIN, I.; PIRES, A. V.; MENDES, C. Q.; OLIVEIRA JÚNIOR, R. C. Substituição de feno de "Coastcross" (Cynodon sp.) por casca de soja na alimentação de borregas confinadas. Ciência Rural, Santa Maria, v. 37, n. 4, p. 1073-1078, 2007.

NATIONAL RESEARCH COUNCIL - NRC. Nutrient requirements of sheep. 6. ed. Washinton, D.C., 1985. 99 p.

7. ed. rev. Washinton, D.C., 2001. 381

p.

. Nutrient requirements of small ruminants: sheep, goats, cervids, and new world camelids. Washinton, D.C, 2007. 408 p.

PEREIRA, E. S.; ARRUDA, A. M. V.; MIZUBUTI, I. Y.; SILVA, L. D. F. da. Consumo voluntário em ruminantes. Semina: Ciências Agrárias, Londrina, v. 24, n. 1, p. 191196, 2003.

PIRES, C. C.; ARAÚJO, J. R.; BERNARDES, R. A. C.; LANES, R. C.; JUNGES, E. R. V. Desempenho e características da carcaça de cordeiros de três grupos genéticos abatidos ao mesmo estágio de maturidade. Ciência Rural, Santa Maria, v. 29, n. 1, p. 155-158, 1999.
RESTLE, J.; VAZ, F. N. Confinamento de bovinos puros e cruzados. In: LOBATO, J. F. P.; BARCELLOS, J. O. J.; KESSLER, A. M. (Ed.). Produção de bovinos de corte. Porto Alegre: EDIPUCRS, 1999. p. 141-168.

RIBEIRO, L. A. O. Sobrevivência e desempenho de cordeiros do período perinatal ao desmame. In: SENAR. Programa de treinamento em ovinocultura. Porto Alegre: FARSUL/SENAR, 1996. p. 100-108.

SENGER, C. C. D.; KOZLOSKI G. V.; BONNECARRERE SANCHEZ, L. M.; MESQUITA, F. R.; ALVES, T. P. ; CASTAGNINO, D. S. Evaluation of autoclave procedures for fibre analysis in forage and concentrate feedstuffs. Animal Feed Science and Technology, Amsterdam, v. 146, n. 1-2, p. 169-174, 2008.

SILVA, D. J. Análise de alimentos: métodos químicos e biológicos. Viçosa: UFV, 1998. 165 p.

STATISTICAL ANALYSIS SYSTEM - SAS. Statistical analysis system user's guide: statistics. Version 8.2, Cary: SAS Institute, 2001. 1686 p.

SUSIN, I.; ROCHA, M. H. M.; PIRES, A. V. Efeito do uso de bagaço de cana-de-açúcar in natura ou hidrolisado sobre o desempenho de cordeiros confinados. In: REUNIÃO ANUAL DA SOCIEDADE BRASILEIRA DE ZOOTECNIA, 37., 2000, Viçosa, MG. Anais... Viçosa: UFV, 2000. CD-ROM.

ZAMBOM, M. A.; SANTOS, G. T.; MODESTO, E. C.; ALCALDE, C. R.; GONÇALVES, G. D.; SILVA, D. C. da; SILVA, K. T. da; FAUSTINO, J. O. Valor nutricional da casca do grão de soja, farelo de soja e milho moído e farelo de trigo para bovinos. Acta Scientiarum, Maringá, v. 23, n. 4, p. 937-943, 2001. 